\title{
Designing Energy-Aware Adaptive Routing for Wireless Sensor Networks
}

\author{
Jing Zhou \\ School of Electronics and Computer Science \\ University of Southampton \\ Southampton, SO17 1BJ, UK \\ Email: jz@ecs.soton.ac.uk
}

\author{
David De Roure \\ School of Electronics and Computer Science \\ University of Southampton \\ Southampton, SO17 1BJ, UK \\ Email: dder@ecs.soton.ac.uk
}

\begin{abstract}
Many energy-aware routing protocols take into account the residual battery power in sensor nodes or/and the energy required for transmission along the path. In the deployment of an environmental sensor network, we observed that applications may also impose requirements on routing, thus placing higher demands on protocol design. We demonstrated our approach to this issue through FloodNet, a flood warning system, which uses a predictor model developed by environmental experts to make flood predictions based on readings of water level collected by a set of sensor nodes. Because the model influences the node reporting frequency, we proposed the FloodNet adaptive routing (FAR) which transmits data across nodes with ample energy and light reporting tasks whilst conserving energy for others low on battery power and heavily required by the monitoring task. As a reactive protocol, FAR is robust to topology changes due to moving obstacles and transient node failure. We evaluate the FAR performance through simulation, the result of which corresponds with its anticipated behavior and improvements.
\end{abstract}

Keywords-Adaptive sampling, energy awareness, environmental sensor networks.

\section{INTRODUCTION}

Wireless sensor networks (WSNs) consist of a number of sensor nodes equipped with sensing, data processing and communications capabilities for monitoring purposes. As microelectronic devices, these nodes have limited supply of energy. However, most WSN applications require very long unattended periods of operation. Also, where hazardous conditions limit human access, replenishment of power resources or replacement of nodes becomes impossible. In a multihop WSN, the malfunctioning of any node may lead to topology changes, or in worse cases, degraded network performance. Efficient energy conservation and management techniques are therefore of primary importance. In some applications, such as ours, the energy replenishment may be at its lowest precisely when the sensor readings are needed with highest priority.

To extend WSN lifetime, many routing protocols have been proposed with energy awareness being an essential design consideration. These schemes involve selecting the optimal path between a data originator and a data collecting unit. The selection criteria take into account the available power in nodes or the energy required for transmission in the links along the path [1]. Some other optimality criteria include hop count, delay, forwarding cost, etc. WSNs may differ depending on application areas as the latter would impose domain-specific requirements on design and management. These requirements should not be underestimated especially if they have a direct impact on the behavior of sensor nodes. Consequently, the resulting routing protocol should have more complex optimality criteria for path selection, coupling application requirements with energy conditions in the network.

We illustrate our approach through FloodNet, a flood early warning system, which deploys a set of intelligent sensor nodes and uses a predictor model [2] to make flood predictions based on sensor readings. The research focus is to leverage the need for timely data and the need to conserve battery power. As a result, the system is made adaptive- the behavior of sensor nodes (i.e. the reporting frequency) varies according to application needs imposed upon by the predictor model. Reducing activity of individual sensor nodes and minimizing the data volume required help prolong network lifetime (definition in Sect. III-C). Moreover, FloodNet achieves energy awareness through its routing algorithm: by carefully routing messages across nodes with ample energy and light reporting tasks, the FloodNet adaptive routing (FAR) can conserve energy for sensor nodes low on battery power or/and heavily required by the monitoring task. Delivery of data messages carrying sensor readings has no dependency on any specific sensor node and the optimal path is computed on demand. Hence, FAR is robust to topological changes due to transient node and link failure.

In our study, nodes are not mobile and the network topology is static. We assume constant transmission power for sensor nodes. The energy consumption during idle time is not included in design and the simulation as we assume a situation under which the energy consumption due to interest diffusion, neighbor status maintenance and data report delivery (see Sect. III) is dominant. Note that due to the inherent nonscalability of the flooding technique, the proposed solution in its current form may not be scalable enough for direct application to large scale sensor networks.

The remainder of the paper is structured as follows. The FloodNet scenario is described in Sect. II and the design of FAR is described in Sect. III. In Sect. IV we evaluate the FAR performance. Section V discusses the difference between FAR and other related efforts on energy aware routing. Finally, we outline some conclusions and future work in Sect. VI. 


\section{The FloodNet SCEnARIO}

The FloodNet project at University of Southampton is currently investigating the use of pervasive computing and Grid computing to provide early warning of possible flood. When a flood occurs, there is a clear correlation between the cost of flood damage and both the water depth and the amount of advance warning time given. By deploying wireless sensor nodes on the floodplain, FloodNet aims to offer better data to a flood predictor model which is used to make flood predictions, and to improve warning times. Sensor nodes collect information about water level which is feed through a message broker, hosted by a gateway, back to University of Southampton. The incoming data is stored on a grid and will be further utilized by the predictor model (see Fig. 1).

The project has deployed a set of intelligent sensor nodes at a stretch of river on the east coast of the UK, chosen as a test site on the basis of its tidal behavior (see Fig. 2). Sensor nodes are battery-powered, with solar cells attached. Hence, the sensor network will be in face of energy shortage if the solar cells have not recharged the battery for a long period of time in cloudy and winter conditions during which the battery voltage drops significantly. This creates a challenge for the design of the network communication protocol, i.e. to best accomplish the monitoring and information dissemination task $^{1}$ whilst delaying the occurrence of energy depletion.

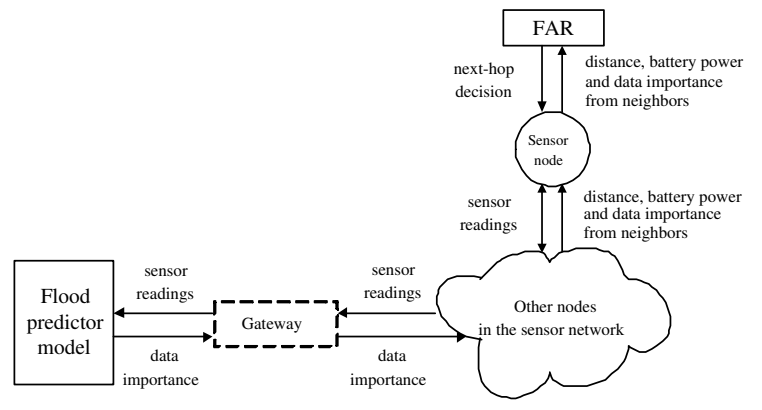

Fig. 1. The FloodNet information flow

The need to conserve battery power for sensor nodes, however, conflicts with the need for timely data, as the more data is sent the more energy is consumed. To prolong the lifetime of the sensor network, sensor nodes must enter into periods of reduced activity when running on low battery voltage [3]. The FloodNet system is therefore made adaptive in which the reporting rates of sensor nodes vary according to need. We have the flood predictor model carry out extensive processing in a short period of time (currently 1 hour). Upon each model iteration ${ }^{2}$ the network changes its behavior, altering the reporting rates for each node in the following model iteration according to the data importance placed upon it by the predictor model. The data importance reflects how important the data from a specific sensor node is.

\footnotetext{
${ }^{1}$ It is measured by success ratio as defined in Sect. IV.

${ }^{2}$ The model iteration refers to the time period during which the predictor model processes sensor readings collected in the previous iteration.
}

The greater the importance value, the higher the reporting rate. The adaptability of reporting rates enables closer monitoring to be achieved in anticipation of possible flood events. The data importance may differ from one node to another because the location of nodes varies from one another-some are deployed in the channel while others are on the floodplain, and therefore the data collected from each node reflects the unique feature of water depth at the region where the node is situated. Sensor nodes will periodically switch on sensors and transmitters, take readings of water depth, and transmit the data at periodic time intervals. The diversity in behavior of nodes requires load balancing as sensor nodes with ample battery and a low reporting rate will encounter energy depletion far behind those with lower battery power and a high reporting rate.

FloodNet relies on the cooperative effort of all nodes to disseminate information across the network. The communication protocol therefore should be robust to the failure of sensor nodes and transmission medium, thus ensuring the functioning of the sensor network. We summarize the main objectives of FAR as follows and describe it in the following section:

- To best accomplish the monitoring and information dissemination task of the sensor network.

- To delay the energy depletion which causes data loss.

- To conserve the battery of nodes with important data.

- To be robust to the failure of sensor nodes and transmission medium.

\section{The Floodnet Adaptive Routing}

This section describes the main components of FAR: interest diffusion, neighbor status maintenance and the FloodNet adaptive routing algorithm.

\section{A. Interest Diffusion}

The environmental requirement, or interest, is diffused into the sensor network from the gateway through a flooding technique. Its description consists of a list of data importance placed upon each node the value of which is within the range $[0,1]$. The gateway node broadcasts an interest message to all neighbors on an hourly basis. An interest message comprises the unique identifier and the residual battery power of the sender, the message type, the distance of the sender from the gateway (in the number of hops), and a list of data importance.

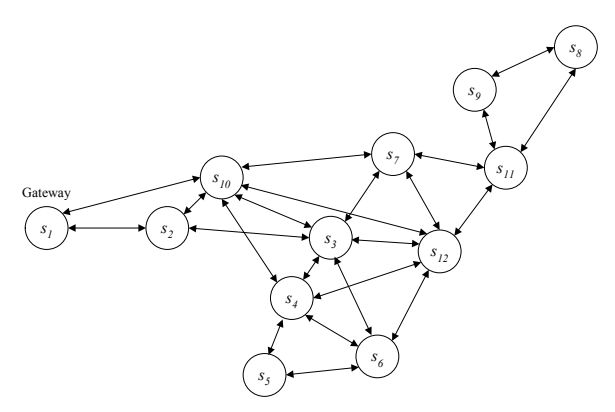

Fig. 2. Interest Diffusion in FloodNet

Each node maintains an interest cache and a neighbor table. If an incoming message contains a fresh interest, then a node 
keeps a copy in cache. For messages regarding the same interest but from distinct senders, the node extracts related information to fill in its neighbor table, replaces the sender identifier with its own identifier, and increases the distance in the interest message by 1 . Only the first message of an interest will be further broadcast to others within the node's wireless transmission range. This process is carried out recursively to ensure that each node will be notified of such an interest. The reporting rate for the recipient node is derived from the data importance. There are currently three different reporting rates allowed for sensor nodes: once per hour, twice per hour, and three times per hour. We apply a straightforward function to convert a data importance $(t)$ to a certain reporting rate $(r)$.

$$
r= \begin{cases}1 & 0 \leq t<0.33 \\ 2 & 0.33 \leq t<0.66 \\ 3 & 0.66 \leq t \leq 1\end{cases}
$$

TABLE I

THE NEIGHBOR TABLE OF NODE $s_{5}$

\begin{tabular}{|c|c|c|c|c|}
\hline node & distance & battery & data importance & link cost \\
\hline$s_{4}$ & 2 & 0.98 & 0.90 & $e_{5,4}$ \\
\hline$s_{6}$ & 3 & 0.99 & 0.60 & $e_{5,6}$ \\
\hline
\end{tabular}

The first message of an interest that a node receives is regarded as indicating the optimal path from the gateway. The following messages are characterized by a distance equal and greater than that in the first and reveal the sub-optimal paths. Table I illustrates the neighbor table of node $s_{5}$ in which the information about its neighbors has been filled in. The residual battery power of neighbors will be regularly updated through neighbor status maintenance as presented in the following section. The link cost is assumed to be known to the involved pair of sensor nodes.

\section{B. Neighbor Status Maintenance}

The neighbor status is captured in each entry of the neighbor table. The information about the residual battery power of neighbors in the neighbor table is refreshed when nodes broadcast their up-to-date status. The frequency on which a node broadcasts its information is determined by the accuracy requirement of the component that will utilize the information. As presented later the FAR algorithm takes into account the up-to-date status of neighbors to decide the optimal path for routing (see Sect. III-C), the frequency of local broadcast should therefore correspond with the reporting rate of nodes because routing is needed once reporting activities commence.

The three reporting rates allowed for FloodNet nodes indicate that local broadcast could occur at the 20th, 30th, 40th, and 60th minute of each model iteration (lasts for 1 hour), and ideally it should happen prior to the start of any reporting activity. Nodes receive update on neighbor status and modify their neighbor table wherever necessary.

\section{Adaptive Routing Algorithm}

The problem in existing energy aware routing protocols is that they try to discover an optimal path and then frequently use the optimal path for every communication, which leads to rapid energy depletion of the nodes on the path. To overcome this problem, Shah and Rabaey [4] proposed an energy aware routing protocol which maintains a set of paths instead of one optimal path and uses one routing path with a certain probability (related to energy consumption on the path) at all times to increase network lifetime.

The flood predictor model, as mentioned earlier, controls the behavior of a sensor node by assigning a data importance for it and the reporting rate of the node is then derived from such a data importance. With no regard of energy consumed in routing messages, one sensor node with a higher reporting rate will consume its battery power faster than another with a lower reporting rate. This application specific feature makes most energy aware routing protocols less feasible for achieving a longer network lifetime as they assume an identical behavior for reporting activities of all nodes.

Prior to presenting the routing algorithm, we feel obliged to introduce the concept of the network lifetime which is closely associated with the routing protocol design and will be constantly referred to in the rest of the paper. There exist various definitions for network lifetime, for example [5] defines it as the time until the network partition occurs due to battery depletion whilst [6] models the network lifetime as the earliest time that a message cannot be sent. The main objective of FloodNet is to best accomplish the monitoring and information dissemination task of the sensor network. Therefore energy conserving is intended to save energy for data message generation (at nodes), and for data message routing (back to the gateway). Network partition does not necessarily lead to the failure of data message delivery. Hence the network lifetime in FloodNet is defined to be the first time at which data messages cannot be generated or get lost due to energy depletion. If the battery level of a node is so low that it cannot transmit a data message at full power, then we consider the messages that the node holds but has not sent, are lost.

We propose an adaptive routing protocol for FloodNet which takes into account a number of factors to make a decision on forwarding data messages. We use a metric, weight, to denote the degree that a sensor node should be chosen as a next-hop node in data forwarding. Ideally, the greater the weight is, the more likely it should be selected as the next-hop node. Suppose $s_{0}$ is going to transmit a data message to another node to route through and $s_{1}$ is one of its neighbors. Let $b_{1}$ represent the residual battery power of $s_{1}, e_{0,1}$ be the link cost for transmission of the data message between $s_{0}$ and $s_{1}$, and $t_{1}$ denote the data importance of $s_{1}$. Let $c_{1}$ denote the transmission capability of $s_{1}$ which is defined as the ratio of the residual battery power of $s_{1}$ to link cost $e_{0,1}$, i.e. $c_{1}=\frac{b_{1}}{e_{0,1}}$.

We propose the following form for the weight of sensor node $s_{1}$ with respect to $s_{0}$ :

$$
w_{0}^{1}=\frac{c_{1}}{\beta^{t_{1}}} .
$$


where the tunable parameter $\beta \geq 1$. The discussion about the best value range for $\beta$ can be referred to in Sect. IV-B.

The weight function has been carefully chosen to achieve the third objective of adaptive routing (see Sect. II). It produces a higher weight which means more chances of being used as a router for data transmission for nodes with ample battery power (or leading to a low energy consumption path) and a light reporting task, whilst giving a lower weight to those with a lower level of battery power (or leading to a high energy consumption path) and a heavy reporting task.

Further, the weight function can prevent the battery power of some nodes from being drained much earlier than that of others. Suppose $s_{0}$ has two neighbors $s_{1}$ and $s_{2}$ with $t_{2}>t_{1}$. If $c_{2}$ is far greater than $c_{1}$, i.e. $c_{2}-c_{1}>C_{\text {threshold }}$, it is reasonable to choose $s_{2}$ as the next-hop node especially when $s_{1}$ has very little residual energy or $s_{1}$ is associated with a very high link cost. With the weight function, $s_{2}$ will be selected as the next-hop node if the following inequality holds:

$$
c_{2}>c_{1} * \beta^{t_{2}-t_{1}} \geq c_{1} .
$$

In the cases where a few nodes share the same weight while being associated with different transmission capabilities and data importance, we apply the following rules:

- If nodes have the same weight and different importance, $s_{0}$ shall choose the one with the least importance.

- If nodes have the same weight and the same importance, $s_{0}$ shall choose the one with the least distance.

- If nodes share the same priority, importance, and distance, then $s_{0}$ shall randomly choose one.

We present the adaptive routing algorithm as follows.

1) A node forwards a data message to a neighbor with the highest weight among all neighbors closer to the destination.

2) If no such neighbors exist, the node sends the message to a neighbor with the highest weight among all that are of the same distance from the destination.

3) If all neighbors are farther from the destination, the node sends the message to one with the highest weight. Otherwise, the message is dropped.

4) Step 1,2 and 3 are repeated until the data message reaches the gateway, or is dropped out.

Each data message carries the sender identifier, the receiver identifier, the message type, the sensor readings and a list of visited nodes. Hence, forwarding loops can be avoided as a sender will always check the list of visited nodes in the data message before retransmitting it.

\section{Performance Evaluation}

\section{A. The Simulation}

FAR is an upper layer (network) protocol that assumes a collision-free, multiple retry MAC layer. We constructed a simulator that implemented the basic FAR protocol. In addition to a number of parameter choices in the protocol, we can also vary certain factors in simulation such as the number of sensor nodes, the pattern of power consumption of nodes, and the frequency of reporting activities of sensor nodes. As well as validating the algorithm, the simulator provided a tool for engagement with environmental experts who were specifying node sampling behaviors.

The basic parameters were chosen to model an ad hoc network consisting of 12 nodes with 1 of them (i.e. $s_{1}$ ) residing on the gateway. The initial energy for all nodes (except $s_{1}$ which is assumed to have ample energy at all times) is 1 unit. The battery consumptions of full power transmitting and receiving are 0.005 unit and 0.0005 unit. Each model iteration is simulated to last for 1 hour. Sensor nodes may generate and send reports (wrapped in data messages) at the 20th, 30th, 40th and 60th minute of each model iteration which correspond to the 4 time units of 1 simulated hour.

We measured the success ratio which is defined as the ratio between the number of data messages successfully received by the gateway to the total number of data messages that should be generated by all nodes (except $s_{1}$ ). We also measured the network lifetime which is equivalent to the time unit at which data messages cannot be generated or get lost due to energy depletion. The node operational time is defined as the time unit at which the energy of any node is depleted.

For exploring the robustness of FAR, link failure was simulated by random failure of links in each model iteration and the link failure rate is defined as that probability. Furthermore, the Energy Aware Routing protocol (EAR) [4] was implemented by the simulator as we will evaluate the performance of FAR against EAR under conditions involving different topologies (mostly randomly generated) and network size. The weighting factors used in the experiments on EAR are $\alpha=1$ and $\beta=$ 50, as used in [4]. To study the performance increase of FAR over EAR as a function of network density, we also generated sensor fields (30 units by 30 units) in which different number of nodes are randomly placed. The number of nodes is ranging from 8 to 24 nodes in increments of 4 nodes and each node has a transmission range of 15 units.

\section{B. Results}

We executed 20 runs of the simulator for each of different protocols and of a number of different tunable weight $\beta$ (in FAR) in the simulated ad hoc network ${ }^{3}$.

TABLE II

FAR WITH DIFFERENT $\beta$ IN RANDOM TOPOLOGIES

\begin{tabular}{|l|r|r|r|r|r|}
\hline & FAR(1.0) & FAR(1.0005) & FAR(1.005) & FAR(1.05) & FAR(1.5) \\
\hline NL & 41.10 & 41.70 & 41.30 & 41.20 & 40.85 \\
\hline NOT & 41.05 & 41.35 & 40.95 & 40.90 & 40.40 \\
\hline
\end{tabular}

Table II reveals that a $\beta$ value approaching 1.0 (e.g. 1.0005 in our simulation) always leads to a very good performance, i.e. the longest network lifetime (NL) and node operational time (NOT). Equation 3 shows that $\beta$ is essentially a knob which adjusts the threshold (i.e. $\beta^{t_{2}-t_{1}}$ ) beyond which one node $\left(s_{1}\right)$ with less important data will be discarded in favor of

\footnotetext{
${ }^{3}$ It should be noted that $s_{1}$ is situated on the gateway and therefore all simulation results presented in this section do not include data of $s_{1}$.
} 


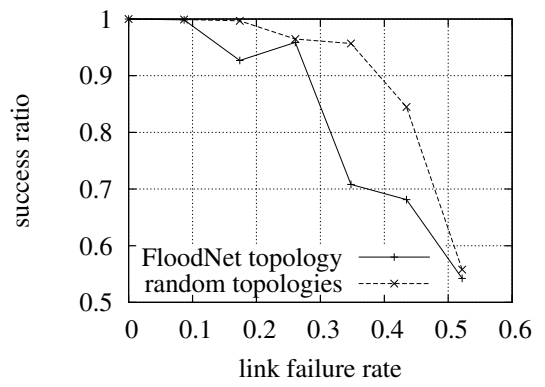

Fig. 3. Success ratio for different link failure rates, $\beta=1.0005$

another $\left(s_{2}\right)$ with more important data and much more battery power. We believe that a small $\beta$ will enable the protocol to have more choices in selecting the next-hop node. This is because the nodes that satisfy Equation 3 with a small $\beta$ will be more than those that satisfy the same equation with a very large $\beta$. The simulation result demonstrates that such a small $\beta$ prevents the energy of some nodes being depleted far earlier than that of others by having them consume battery power in response to needs. The best value for $\beta$, however, may need to be tuned to the particular example scenario.

Figure 3 plots the success ratio as a function of the link failure rate in an ad hoc network of different topologies. FAR delivers a higher success ratio in random topologies than in the FloodNet topology. In the FloodNet topology, the success ratio is above $90 \%$ for the link failure rate up to $28 \%$. In a small-sized ad hoc network like FloodNet, the functioning of certain links such as $s_{0} \leftrightarrow s_{2}$ and $s_{0} \leftrightarrow s_{10}$, is more crucial to a high success ratio than that of others, as no data messages can be successfully delivered to the gateway if they both fail. In random topologies, when $39 \%$ links fail, the success ratio remains above $90 \%$. We believe the robustness of FAR is attributed to its routing algorithm as the latter always tries to find alternative forwarding neighbors even if there is a hole (i.e. no nearer neighbor exists) that could be caused by the failure of either links or nodes.

TABLE III

NETWORK LIFETIME AND NODE OPERATIONAL TIME FOR FAR AND EAR

\begin{tabular}{|l|c|c|c|c|c|c|c|c|}
\hline \# nodes & \multicolumn{4}{|c|}{12} & \multicolumn{4}{c|}{24} \\
\hline topology & \multicolumn{2}{|c|}{ FloodNet } & \multicolumn{2}{c|}{ random } & \multicolumn{2}{c|}{ FloodNet-like } & \multicolumn{2}{c|}{ random } \\
\hline protocol & EAR & FAR & EAR & FAR & EAR & FAR & EAR & FAR \\
\hline NL & 29.75 & 32.55 & 39.20 & 41.70 & 15.85 & 16.20 & 26.65 & 30.30 \\
\hline NOT & 29.45 & 32.25 & 38.85 & 41.35 & 15.80 & 16.20 & 26.05 & 29.80 \\
\hline
\end{tabular}

Table III presents NL and NOT that FAR ${ }^{4}$ and EAR can achieve. FAR outperforms EAR in all cases which differ in the number of nodes in the network. In the FloodNet topology with 12 nodes, FAR extends NL by $9.4 \%$ and NOT by $9.5 \%$ over EAR. Both protocols deliver a longer NL and a longer NOT in random topologies than in the FloodNet topology.

\footnotetext{
${ }^{4}$ For simulations carried out in random topology, $\beta=1.0005$, whereas for others in the FloodNet topology, we observed the best value for $\beta$ is 1.0001 and we used this value in related simulations.
}

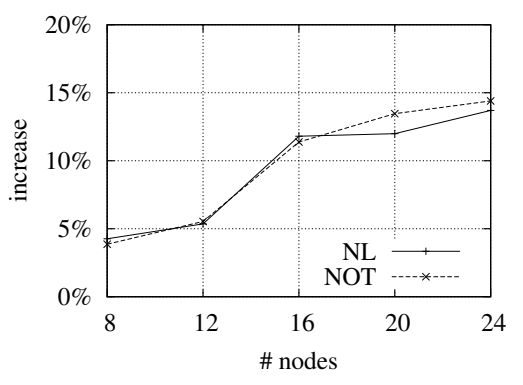

Fig. 4. The effect of network density on performance increase introduced by FAR over EAR in random topologies

This is because the outgoing links are more evenly distributed among sensor nodes in the random topologies than in the FloodNet topology. As a consequence, the probability that the energy of nodes closer to the gateway is depleted much earlier than that of others, is reduced.

Figure 4 shows the effect of network density on the performance increase of FAR over EAR in random topologies. In a very low density network (with 8 nodes), FAR achieves a little increase (around 4\%) in both NL and NOT. As the network density becomes higher, FAR will deliver a better performance. An increased network density means more neighbors per node on average. Hence, FAR will have more candidate nodes to choose from to determine a desirable routing path. By examining the simulation trace we found that the improved performance by FAR is obtained at the cost of more energy consumption. This is because the routing decision may have to involve a longer path in order to extend network lifetime whilst satisfying the environmental monitoring requirement, as also observed by Singh et al. [7].

\section{RELATED WORK}

In this section, we compare existing energy aware routing protocols with FAR. To the best of our knowledge, none of the related work described in this section examines the impact of diverse reporting rates on protocol design.

Stojmenović and Lin [8] proposed power-aware localized routing which only requires localized routing information to minimize energy consumption and extend battery's worse case lifetime. The absence of mechanisms to provide node location information makes us unable to directly apply their approach to FloodNet. Other protocols using location information of nodes include GEAR [9] which achieves a longer network lifetime by balancing energy usage across the network ${ }^{5}$.

To maximize network lifetime, Chang and Tassiulas [5] proposed formulating the routing problem in WSNs consisting of static nodes as a linear programming problem for constant data rates as well as arbitrary processes. Their major assumption is that global network information is available for decision making on routing, which was also shared by [6]. Hence, each origin node of a commodity can calculate the shortest

\footnotetext{
${ }^{5} \mathrm{He}$ et al. also argued in SPEED [10] that balanced energy consumption would increase network lifetime.
} 
cost path to its destination node. However, this is not suitable for localized routing algorithms like FAR.

LEACH [11], PEGASIS [12] and the Energy Aware Routing [4] extend network lifetime by evenly distributing the energy load among all nodes. They assumed a fixed sampling and reporting rate for all nodes. Although there are superficial similarities between LEACH and FAR, and specifically LEACH also considers non-uniform energy consumption of nodes, e.g. cluster-heads often consume more energy for data forwarding, LEACH essentially achieves a longer network lifetime by evenly dissipating energy among nodes whilst FAR does so by enabling nodes to consume energy according to need.

Schurgers et al. [13] proposed using topology management techniques to coordinate the sleep transitions of all nodes whilst ensuring adequate network connectivity. The sensor network was assumed to be in the monitoring state during most of its lifetime. This scenario also motivated [14], an energyefficient MAC protocol which reduces the waste of energy from collision, overhearing, control package overhead and idle listening. In contrast, nodes in FloodNet are required to report data at different but more frequent rates.

Pulse [15] was designed for multi-hop wireless infrastructure access to mobile users which utilizes a periodic flood initiated at gateways to provide routing and synchronization information to the network. Substantial energy savings can be achieved by using the synchronization information to allow idle nodes to power off their radios for most of the time when they are not required for packet forwarding. The Pulse flood proactively maintains a route from all nodes in the network to the infrastructure access node. This is in contrast with FAR which selects the routing path on-the-fly as data messages traverse the network and thus is robust to temporary failure.

\section{CONCLUSions AND Future Work}

We believe ignoring requirements imposed upon by applications may prevent energy-aware routing protocols from being most desirable, and have demonstrated our solution by developing an energy-aware adaptive routing protocol, FAR, for FloodNet and related projects. FAR takes into account the distinct behavior of individual nodes and uses the weight function as well as a set of rules in selecting a neighboring node to forward data messages. It allows messages to be carefully routed across sensor nodes with ample energy and light reporting tasks whilst conserving energy for those which have low energy level and heavy reporting tasks. FAR is robust to temporary failure as the routing algorithm will always make an effort to find alternative forwarding neighbors. Simulation results confirm the anticipated behavior of FAR and its improvements over EAR in the simulated environments. FAR has achieved all of its design objectives.

As introduced in Sect. III-A, we currently employ a straightforward function to convert a data importance to one of the three reporting rates allowed. Equation 2 shows that, given the same transmission capability $c$, weight $w$ is more sensitive to variations of data importance $t$ when the latter is small. This indicates that we could use alternative conversion functions if necessary. We will need to investigate the appropriate conversion functions by comparing predictions given by the flood predictor model with real water depth measurements for which the predictions is made to adjust the ranges for reporting rates, so as to provide more precise flood warnings. Further, we will explore how FAR works with sensor nodes that have the ability to control the power of their radio transmissions.

\section{ACKNOWLEDGEMENT}

FloodNet is funded by the UK Department of Trade and Industry under the Next Wave Programme, as part of the EnviSense Centre for Pervasive Computing in the Environment. We also acknowledge the input of the Equator project (EPSRC Grant GR/N15986/01).

\section{REFERENCES}

[1] I. F. Akyildiz, W. Su, Y. Sankarasubramaniam, and E. Cayirci, "A Survey on Sensor Networks," IEEE Communications Magazine, vol. 40, no. 8, pp. 102-114, Aug. 2002.

[2] J. Neal, P. Atkinson, and C. Hutton, "Real-time flood modelling using spatially distributed dynamic depth sensors," in Geosensornetzwerke von der Forschung zur praktischen Anwendung, C. Brox, A. Krüger, and I. Simonis, Eds., vol. 23. Institut für Geoinformatik, Universität Münster, Germany: IfGI prints, 2005, pp. 147-159.

[3] A. Sinha and A. Chandrakasan, "Dynamic power management in wireless sensor network," IEEE Design \& Test of Computers, vol. 18, no. 2, pp. 62-74, Mar. 2001.

[4] R. C. Shah and J. Rabaey, "Energy Aware Routing for Low Energy Ad Hoc Sensor Networks," in Proceedings of IEEE Wireless Communications and Networking Conference (WCNC), vol. 1, Orlando, FL, Mar. 2002, pp. 350-355.

[5] J.-H. Chang and L. Tassiulas, "Maximum lifetime routing in wireless sensor networks," IEEE/ACM TRANSACTIONS ON NETWORKING, vol. 12, no. 4, pp. 609-619, Aug. 2004.

[6] Q. Li, J. Aslam, and D. Rus, "Online power-aware routing in wireless adhoc networks," in Proceedings of the 7th annual international conference on Mobile computing and networking, Rome, Italy, 2001, pp. 97-107.

[7] S. Singh, M. Woo, and C. S. Raghavendra, "Power-aware routing in mobile ad hoc networks," in Proceedings of the 4th annual ACM/IEEE international conference on Mobile computing and networking, Dallas, Texas, USA, 1998, pp. 181-190.

[8] I. Stojmenović and X. Lin, "Power-aware localized routing in wireless networks," IEEE Transactions on Parallel and Distributed Systems, vol. 12, no. 10, pp. 1-12, Oct. 2001.

[9] Y. Yu, R. Govindan, and D. Estrin, "Geographical and Energy Aware Routing: a recursive data dissemination protocol for wireless sensor networks," UCLA Computer Science Department, Technical Report UCLA/CSD-TR-01-0023, May 2001.

[10] T. He, J. A. Stankovic, C. Lu, and T. F. Abdelzaher, "A spatiotemporal communication protocol for wireless sensor networks," IEEE Transactions on Parallel and Distributed Systems, vol. 16, no. 10, pp. 995-1006, Oct. 2005.

[11] W. Heinzelman, A. Chandrakasan, and H. Balakrishnan, "Energyefficient communication protocol for wireless microsensor networks," in Proceedings of the 33rd Hawaii International Conference on System Sciences, vol. 8, Jan. 2000, pp. 3005-3014.

[12] S. Lindsey and C. S. Raghavendra, "PEGASIS: Power-Efficient GAthering in Sensor Information Systems," in IEEE Aerospace Conference Proceedings, vol. 3, Mar. 2002, pp. 1125-1130.

[13] C. Schurgers, V. Tsiatsis, S. Ganeriwal, and M. Srivastava, "Topology management for sensor networks: exploiting latency and density," in Proceedings of the 3rd ACM international symposium on Mobile ad hoc networking and computing, Lausanne, Switzerland, 2002, pp. 135-145.

[14] W. Ye, J. Heidemann, and D. Estrin, "An energy-efficient mac protocol for wireless sensor networks," in Proceedings of IEEE INFOCOM 2002, vol. 21, no. 1, 2002, pp. 1567-1576.

[15] B. Awerbuch, D. Holmer, and H. Rubens, "The pulse protocol: Energy efficient infrastructure access," in Proceedings of IEEE INFOCOM 2004, Hong Kong, China, Mar. 2004. 